
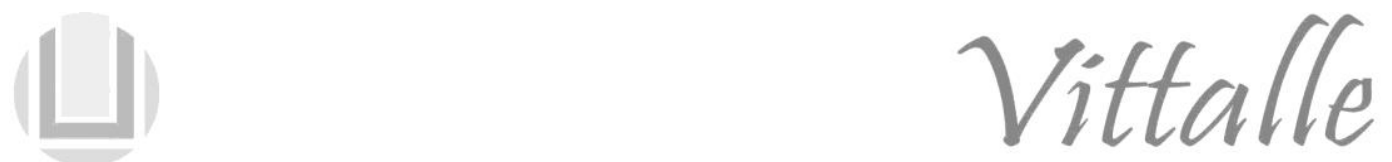

\title{
O tensionamento do acesso na rede de atenção à saúde no extremo sul do Brasil
}

\author{
Cesar Francisco Silva da Costa*, Helena Heidtmann Vaghetti, \\ Nalú Pereira da Costa Kerber, Edison Luiz Devos Barlem
}

Escola de Enfermagem, Universidade Federal do Rio Grande - EENF/FURG, Rio Grande, RS, Brasil

Histórico do Artigo
Recebido em
18/09/2019
Aceito em
04/11/2019

Palavras-chave:
Acesso aos Serviços
de Saúde; Sistema
Único de Saúde;
Gestão em Saúde;
Assistência à Saúde;
Referência e
Consulta

Keywords:

Health Services

Accessibility; Unified

Health System; Health

Management; Delivery

of Health Care;

Referral and

Consultation

\begin{abstract}
RESUMO
Este estudo tem como objetivo identificar outras formas de acesso, que não a pactuada entre os gestores, ao ambulatório do hospital universitário de referência de uma microrregião ao sul do Brasil. Estudo de casos múltiplos integrados, em que a coleta dos dados foi por entrevistas realizadas entre julho de 2015 e julho de 2016, utilizando um roteiro elaborado e adaptado, baseado no questionário proposto por Eugênio Vilaça Mendes, para avaliação do grau de integração da rede de atenção à saúde. As unidades de análise foram as secretarias municipais de saúde de uma microrregião e o hospital universitário de referência. Foram participantes gestores, profissionais de saúde, serviço de regulação em saúde e representantes do controle social. A análise foi por meio da análise textual discursiva e o corpus analisado a partir dos textos produzidos por entrevistas e documentos. Constatou-se a existência de tensionamentos como forma de acesso ao serviço do ambulatório por diversos atores na rede: as exercidas pelos usuários; aos e pelos profissionais de saúde; por e entre os gestores; e pelo controle social. É possível afirmar que os tensionamentos como forma de acessar o serviço de ambulatório do hospital universitário de referência existem e que se faz necessário uma efetiva discussão da rede de atenção à saúde da microrregião, não somente na média complexidade, mas por completo, iniciando na atenção primária à saúde em busca da integralidade da assistência.
\end{abstract}

The tensioning of access in the health care network in the extreme south of Brazil

\section{ABSTRACT}

This study aims to identify different ways of access, other than that agreed among the managers, to the outpatient clinic of the reference university hospital in a microregion in southern Brazil. An integrated multiple case study, in which the data were collected through interviews conducted between July 2015 and July 2016, using an elaborate and adapted script based on the questionnaire proposed by Eugênio Vilaça Mendes, to evaluate the degree of integration of the health care network. The units of analysis were the municipal health office of a microregion and the university hospital of reference. Participants were managers, health professionals, health regulators and agents of social control. The analysis was through the discursive textual analysis and the corpus analyzed from the texts produced by interviews and documents. Results: it was verified the existence of tensions as a form of access to the outpatient clinic by several actors in the network: those performed by users; to and by health professionals; by and between managers; and by social control. It is possible to affirm that the tensions as a way of accessing the reference outpatient clinic of the university hospital exist and that an effective discussion of the health care network of the microregion is necessary, not only in medium complexity, but in full, initiating in primary health care aiming integral care.

\section{Introdução}

Redes de Atenção à Saúde (RASs) é um sistema que busca, deliberadamente, no plano de sua institucionalidade, aprofundar e estabelecer padrões estáveis de inter-relações. Para Mendes (1), é imprescindível incorporar como ponto central dessa RAS o critério de acesso, onde o desenho das redes de atenção à saúde faz-se combinando, dialeticamente, de um lado, economia de escala e qualidade dos serviços e, de outro, o acesso aos serviços de saúde.

\footnotetext{
*Autor correspondente: hspcesar@furg.br (Costa C. F. S.)
} 
O conceito de acesso pode variar à medida que as sociedades evoluem e novas necessidades surgem. E por esta evolução, atualmente, o acesso à saúde é cada vez mais discutido em termos de justiça social e de equidade (2).

Na Atenção Primária à Saúde (APS), o acesso pode ser relacionado com as diversas possibilidades de adentrar aos serviços de saúde, estando estas implicadas quanto à localização da unidade de saúde, à disponibilidade de horários e aos dias de atendimento, com a possibilidade de atendimento a consultas não agendadas e a percepção da população quanto à adequação do acesso ao serviço (3).

Uma APS de qualidade, como parte integrante das RASs, orienta-se por eixos estruturantes denominados atributos. Entre os atributos essenciais temos: a atenção ao primeiro contato, a longitudinalidade, a integralidade e a coordenação. Entre os atributos derivados: a orientação familiar e comunitária, e a competência cultural $(3,4)$.

No estado do Rio Grande do Sul (RS) Brasil, os municípios do Rio Grande/RS, São José do Norte/RS, Santa Vitória do Palmar/RS e Chuí/RS em conjunto, integram geograficamente a Microrregião Litoral Lagunar do Rio Grande do Sul $(5,6)$.

$\mathrm{Na}$ busca de identificar características da rede de saúde na Microrregião Litoral Lagunar do RS, realizou-se um Estudo de Caso com o objetivo de identificar outras possíveis formas de acesso, que não a pactuada entre os gestores, ao ambulatório do hospital universitário de referência para a média complexidade na microrregião.

\section{Materiais e métodos}

Trata-se de um artigo oriundo da tese de doutorado em enfermagem, intitulada " $\mathrm{O}$ acesso ao ambulatório do Hospital Universitário da Universidade Federal do Rio Grande na Rede de Atenção à Saúde da Microrregião Litoral Lagunar do Rio Grande do Sul: estudo de caso com gestores, profissionais e controle social" (7).

Foi realizado um Estudo de Casos Múltiplos Integrados (8), em que a metodologia utilizada para a análise foi a Análise Textual Discursiva (9) e o corpus foi analisado a partir do conjunto de textos produzidos por entrevistas (Evidência 1) e documentos (Evidência 2).

As Unidades Integradas de Análise foram as Secretarias Municipais de Saúde dos municípios da Microrregião Litoral Lagunar do Rio Grande do Sul e o Hospital Universitário Dr. Miguel Riet Correa Jr., da Universidade Federal do Rio Grande (HU/FURG), que foi considerado no estudo como Unidade Integrada de Análise 2, em decorrência de ser referência em nível regional de média complexidade. No HU/FURG, além das entrevistas, foram utilizadas também informações documentais que considerou-se como Evidência 2.

Foram sujeitos do estudo 31 participantes, entre gestores, profissionais de saúde, serviço de regulação em saúde e representantes do controle social. Para contemplar o anonimato e a privacidade dos participantes, foram utilizadas siglas. Esta identificação pode ser melhor visualizada na apresentação do quadro abaixo. 
Quadro 1 - Unidades Integradas de Análise. Participantes e Siglas. Estudo de Casos Múltiplos Integrados. Microrregião Litoral Lagunar do RS. 2016.

\begin{tabular}{|c|c|c|}
\hline $\begin{array}{l}\text { Unidade Integrada de } \\
\text { Análise (UIA) }\end{array}$ & Participante & Siglas \\
\hline \multirow{5}{*}{ Chuí/RS } & Médico Unidade Básica Saúde da Família & MED/ESF/CH \\
\hline & Enfermeira Unidade Básica de Saúde & $\mathrm{ENF} / \mathrm{UBS} / \mathrm{CH}$ \\
\hline & Secretário SMS & $\mathrm{G} / \mathrm{CH}$ \\
\hline & Funcionária Serviço Regulação & $\mathrm{R} / \mathrm{CH}$ \\
\hline & Presidente CMS & $\mathrm{CS} / \mathrm{CH}$ \\
\hline \multirow{5}{*}{$\begin{array}{l}\text { Santa Vitória do } \\
\text { Palmar/RS }\end{array}$} & Médica ESF & MED/ESF/SVP \\
\hline & Enfermeira ESF & ENF/ESF/SVP \\
\hline & Secretária SMS & G/SVP \\
\hline & Enfermeira Coordenadora ESF & C/ESF/SVP \\
\hline & Presidente CMS & CS/SVP \\
\hline \multirow{10}{*}{ São José do Norte/RS } & Médico ESF Unidade 1 & MED/ESF1/SJN \\
\hline & Médico ESF Unidade 2 & MED/ESF2/SJN \\
\hline & Enfermeira ESF Unidade 1 & ENF/ESF1/SJN \\
\hline & Enfermeira ESF Unidade 2 & ENF/ESF2/SJN \\
\hline & Secretária Adjunta da Saúde & G/SJN \\
\hline & Coordenadora Programas de Saúde & C/PS/SJN \\
\hline & Coordenadora da Atenção Básica & $\mathrm{C} / \mathrm{AB} / \mathrm{SJN}$ \\
\hline & Funcionária Serviço de Regulação & R1/SJN \\
\hline & Funcionário Serviço de Regulação & R2/SJN \\
\hline & Presidente CMS & $\mathrm{CS} / \mathrm{SJN}$ \\
\hline \multirow{8}{*}{ Rio Grande/RS } & Médica ESF Unidade 1 & MED/ESF1/RG \\
\hline & Médica ESF Unidade 2 & MED/ESF2/RG \\
\hline & Enfermeira ESF Unidade 1 & ENF/ESF1/RG \\
\hline & Enfermeira ESF Unidade 2 & ENF/ESF2/RG \\
\hline & Secretária Adjunta da Saúde & G/RG \\
\hline & Coordenadora da ESF & $\mathrm{C} / \mathrm{ESF} / \mathrm{RG}$ \\
\hline & Funcionário Serviço de Regulação & $\mathrm{R} / \mathrm{RG}$ \\
\hline & Presidente CMS & $\mathrm{CS} / \mathrm{RG}$ \\
\hline \multirow{3}{*}{ Hospital Universitário } & Diretora Adjunta & $\mathrm{G} / \mathrm{HU}$ \\
\hline & Coordenadora do Ambulatório & $\mathrm{C} / \mathrm{AMB} / \mathrm{HU}$ \\
\hline & Funcionária Ações de Regulação & $\mathrm{R} / \mathrm{HU}$ \\
\hline
\end{tabular}

Quanto a Evidência 2, estas aparecem na forma de e-mails encaminhados pelo Conselho Municipal de Saúde ao HU e o teor das mensagens versa sobre solicitação de informações quanto a procedimentos cirúrgicos, realização de exames, documentação de referenciamento de usuário para atendimento, solicitação de leitos hospitalares e comunicação de judicialização para obter acesso ao serviço, esse foram identificados entre parênteses "e-mail" seguidos da data do envio.

Após a aprovação pelo Comitê de Ética em Pesquisa da Universidade Federal do Rio Grande (CEPAS/FURG), sob o Parecer CEPAS 17/2015, e realização de um Estudo de Caso-Piloto, conforme recomendado pela metodologia (8), deu-se início a pesquisa.

A coleta dos dados ocorreu entre julho de 2015 e julho de 2016 e foi realizada por meio de entrevistas utilizando um roteiro semiestruturado. Houve o deslocamento do pesquisador até os municípios, sendo as entrevistas gravadas e transcritas. Para acessar os documentos da Evidência 2, foi obtido o consentimento da direção do HU/FURG.

Os itens que compõem o instrumento para a entrevista e que serviram como foco de orientação do entrevistador foram elaborados em parte e adaptados, com base no questionário proposto por Eugênio Vilaça Mendes (1) para avaliação do grau de integração das RASs, construído a partir das concepções teórica e operacional, medido nas dimensões da população, da APS, dos pontos de atenção secundária e terciária, dos sistemas de apoio, dos sistemas logísticos, do sistema de governança e do modelo de atenção à saúde. 


\section{Resultados}

Conforme os relatos obtidos durante as entrevistas, da fonte documental, e realização da análise conforme metodologia de Análise Textual Discursiva (9) foi possível constatar a existência de tensionamentos do sistema por parte dos diversos atores envolvidos, como forma de acesso ao serviço de saúde na RAS da Microrregião Litoral Lagunar.

É possível identificar, nas falas dos 31 participantes, que entre eles houve $21(67,74 \%)$ que referiram a existência de tensionamentos no acesso aos serviços de saúde. Esta relação pode ser melhor visualizada na apresentação do quadro abaixo.

Quadro 2 - Unidades Integradas de Análise. Participantes e Tensionamentos no sistema. Estudo de Casos Múltiplos Integrados. Microrregião Litoral Lagunar do RS. 2016.

\begin{tabular}{|c|c|c|c|c|}
\hline \multirow[b]{2}{*}{$\begin{array}{l}\text { Unidade Integrada de Análise } \\
\text { (UIA) }\end{array}$} & \multicolumn{4}{|c|}{ Tensionamentos no sistema segundo o participante } \\
\hline & $\begin{array}{l}\text { Por ação dos } \\
\text { usuários }\end{array}$ & $\begin{array}{c}\text { Aos/pelos } \\
\text { profissionais de } \\
\text { saúde }\end{array}$ & $\begin{array}{l}\text { Por/entre } \\
\text { os gestores }\end{array}$ & $\begin{array}{l}\text { Pelo controle } \\
\text { social }\end{array}$ \\
\hline \multirow{4}{*}{$\begin{array}{l}\text { Chuí/RS } \\
\text { (CH) }\end{array}$} & MED/ESF/CH & MED/ESF/CH & & \\
\hline & ENF/UBS/CH & & & \\
\hline & & & $\mathrm{G} / \mathrm{CH}$ & \\
\hline & & & $\mathrm{CS} / \mathrm{CH}$ & \\
\hline \multirow{4}{*}{$\begin{array}{l}\text { Santa Vitória do Palmar/RS } \\
\text { (SVP) }\end{array}$} & & MED/ESF/SVP & & \\
\hline & ENF/ESF/SVP & & & \\
\hline & & G/SVP & G/SVP & \\
\hline & & & & CS/SVP \\
\hline \multirow{5}{*}{$\begin{array}{l}\text { São José do Norte/RS } \\
\text { (SJN) }\end{array}$} & MED/ESF1/SJN & & & \\
\hline & ENF/ESF1/SJN & & & \\
\hline & & ENF/ESF2/SJN & & \\
\hline & & & C/PS/SJN & \\
\hline & & & R1/SJN & \\
\hline \multirow{5}{*}{$\begin{array}{l}\text { Rio Grande/RS } \\
\text { (RG) }\end{array}$} & & MED/ESF1/RG & & \\
\hline & & MED/ESF2/RG & & \\
\hline & & ENF/ESF2/RG & & \\
\hline & & & $\mathrm{R} / \mathrm{RG}$ & $\mathrm{R} / \mathrm{RG}$ \\
\hline & CS/RG & & & \\
\hline \multirow{3}{*}{$\begin{array}{l}\text { Hospital Universitário } \\
\text { (HU) }\end{array}$} & $\mathrm{G} / \mathrm{HU}$ & $\mathrm{G} / \mathrm{HU}$ & $\mathrm{G} / \mathrm{HU}$ & \\
\hline & & C/AMB/HU & & \\
\hline & $\mathrm{R} / \mathrm{HU}$ & & $\mathrm{R} / \mathrm{HU}$ & $\mathrm{R} / \mathrm{HU}$ \\
\hline
\end{tabular}

Entre as ocorrências de tensionamentos, é possível destacar pelo menos quatro: as exercidas pelos usuários; as exercidas aos e pelos profissionais de saúde; as exercidas por e entre os gestores; e as exercidas pelo controle social.

\section{Tensionamentos no sistema por ação dos usuários}

Na Evidência 1, UIA 1, quanto ao tensionamentos no sistema por ação dos usuários, identificaram que, no $\mathrm{CH}$, SVP e SJN, o tensionamento por parte dos usuários é identificado conforme relato dos profissionais de saúde.

\footnotetext{
"Mesmo os brasileiros acessam lá, porque estamos na fronteira e tem cidadania brasileira e uruguaia.". (MED/ESF/CH)

"[...] o cartão SUS eles conseguem fácil. Não tem nada que divida a fronteira [...] tem um parente aqui, dá o endereço e consegue o cartão SUS.". (ENF/UBS/CH)

"Não existe uma regulação ou avaliação eficiente; então as pessoas acabam por políticas partidárias tendo acesso a medicamentos ou a consultas, de passar na frente da fila.". (ENF/ESF/SVP)
} 


\begin{abstract}
“[...]. Escrevi cardiologia urgente e referenciei. Aí ele me perguntou: 'Doutor. quanto tempo vai demorar para eu ser atendido? [...]. E se eu for lá no hospital de cardiologia, dizendo que estou sentindo dor, eles me atenderiam?'. [...] Quer dizer, existe uma deficiência na referência, a gente percebe isso e o paciente também.". (MED/ESF1/SJN)

"Fizemos a nossa parte. Não era urgente, mas a mãe ficou preocupada. O médico viu, prescreveu, mas ela foi lá.". $(E N F / E S F 1 / S J N)$
\end{abstract}

No município do RG o tensionamento é identificado conforme relato do controle social.

“[...] então por que eu vou lá na unidade básica se eu posso ir na Santa Casa? [...] tem alguns casos que, devido à proximidade com Santa Vitória e São José do Norte, o fluxo se confunde. [...] eles vêm consultar aqui sem serem referenciados. (CS/RG)

Os resultados obtidos na Evidência 1, UIA 2: No HU, o tensionamento por parte do usuário é identificado conforme relato do serviço de regulação e pela gestão.

\begin{abstract}
"[...] Como não conseguem através da secretaria, eles fazem contato com o hospital.”. (R/HU)

"Criamos a cartilha do HU, na tentativa de fazer com que o cidadão saiba como e onde procurar atendimento. Ainda assim observamos que eles querem entrar pelo SPA, porque é a forma mais rápida de resolubilidade.". (G/HU)
\end{abstract}

\title{
Tensionamentos no sistema exercidos aos e pelos profissionais de saúde
}

Os resultados obtidos na Evidência 1, UIA 1, quanto aos tensionamentos no sistema exercidos aos e pelos profissionais de saúde.

No $\mathrm{CH}$, esse tensionamento é identificado conforme relato do profissional de saúde. E, em SVP, é identificado conforme relato da gestão e do profissional de saúde.

"É uma configuração que só o Chuí tem. Eles usam o registro de fronteira e aí usa dos dois lados: Brasil e Uruguai. Para nós médicos, facilita; para o sistema de saúde brasileiro, acho que nem tanto.". (MED/ESF/CH)

"Aquele que está precisando mesmo, mas que não briga tanto é o que não consegue saber como funciona, e aí o profissional de saúde orienta a ir à secretaria de saúde.". (G/SVP)

"[...] tem especialidade que é mais rápido; a gente liga para o profissional.”. (MED/ESF/SVP)

Em SJN e em RG é identificado conforme relato dos profissionais de saúde.

"Quando tenho necessidade de alguma especialidade urgente, faço contato e vou ver o que pode ser feito.". (ENF/ESF2/SJN)

"O médico da minha unidade trabalha como pediatra à noite no hospital do Norte. Se tiver algum problema, o médico mesmo resolve.". (ENF/ESF1/SJN)

"A oncologia se consegue ligar e agendar quando tem um diagnóstico. A saúde da mulher também; ligamos e nos casos urgentes se consegue. Os exames laboratoriais são coletados aqui na 
unidade e gestante tem prioridade. Se tem um caso urgente, conversamos com o pessoal e conseguimos encaixar no dia da coleta.". (MED/ESF1/RG)

"[...] urgência oftalmológica que chega ao posto, ligamos para secretaria e eles conseguem uma consulta, pagam uma consulta particular. [...] 'otorrino' as cirurgias têm que ser em Bagé. Tentamos conversar com a secretária da saúde antes dela sair para ver se operava aqui na Santa Casa; não conseguimos.". $(M E D / E S F 2 / R G)$

"[...] para especialidades, sendo urgente, encaminhamos via telefone [...] 'endócrino', até mês passado estava fechada para casos novos. Quando precisamos para paciente diabético descompensado, insulinodependentes, as médicas entram em contato direto com o pessoal da residência, que está lá no serviço de "endócrino"; eles fazem o acolhimento e dão retorno à unidade.". (ENF/ESF2/RG)

Os resultados obtidos na Evidência 1, UIA 2: No HU os tensionamentos exercidos aos e pelos profissionais de saúde é identificado conforme relato da coordenação do ambulatório e da gestão.

"[...] há vezes em que diante do que o paciente está trazendo e do nosso olhar clínico, converso com o médico para compartilhar essa necessidade e então o paciente é atendido como extra. [...], procuro ser bastante criteriosa; então, consigo acessar de forma diferenciada e com responsabilidade quando tenho ciência do caso.". $(\mathrm{C} / \mathrm{AMB} / \mathrm{HU})$

"O HU recebe até de profissionais que não da rede, mesmo quando não somos referência.”. (G/HU)

\section{Tensionamento por e entre os gestores}

Os resultados obtidos na Evidência 1, UIA 1: Quanto ao tensionamento por e entre os gestores, no $\mathrm{CH}$, este tensionamento é identificado conforme relato da gestão e do controle social.

\footnotetext{
"Somos vinte e três secretários municipais e mantemos contatos uns com outros. Criamos via internet um canal e quase que diariamente nos comunicamos e nos ajudamos. Criamos uma rede social. [...].". (G/CH) "A Barra do Chuí é aqui pertinho; pertence a Santa Vitória. Nos ligam da prefeitura de Santa Vitória. Teve um acidente ali. Aí o Chuí leva para Santa Vitória, mas o custo desse atendimento é para o Chuí. O hospital de Santa Vitória cobra do Chuí.". (CS/CH)
}

Em SVP é identificado conforme relato da gestão.
"Quando o sistema oferta consulta que estejam restando de outros municípios, tentamos nos enquadrar. Quando não conseguimos dessa maneira, fazemos contato com os municípios, com outros gestores. Procuramos respeitar todos os sistemas. Mesmo que a nossa referência não seja aquele município, tentamos encaminhar um paciente, através de uma pessoa conhecida que se tenha lá, porque é a vida do paciente que não pode esperar." (G/SVP)

Em SJN é identificado conforme relato da coordenação de programas de saúde e do serviço de regulação. 


\begin{abstract}
"Nossa referência pela PPI é o HU, mas não estão marcando gestante de alto risco. Por um acordo conseguimos mandar para Pelotas. Elas vão de lancha, depois vão até Pelotas de ônibus, pegam um onibus circular da cidade, consultam e voltam. ". (C/PS/SJN)

"A cardiologia pediátrica ou a cirurgia pediátrica pela entrada no sistema é o G-MUS, vai para FURG e demora. Se mandar pelo sistema de Porto Alegre, a consulta é na outra semana, bem rápido.". (RI/SJN)
\end{abstract}

Em RG é identificado conforme relato do serviço de regulação.

"As secretarias de saúde dos municípios entram em contato com a de Rio Grande e organizamos de acordo com a demanda. Não podemos passar por cima do estabelecido pela secretaria do Estado, mas sendo prioridade, eles mandam e-mail para o nosso serviço, e também pedem pelo sistema informatizado.". $(R / R G)$

Os resultados obtidos na Evidência 1, UIA 2: No HU, o tensionamento por e entre os gestores é identificado conforme relato da gestão e do serviço de regulação.

"Como gestora, aprendi o quão difícil é referenciar. Nem sempre pelas vias de direito conseguimos. Há vezes em que precisamos falar diretamente com quem está acima da nossa capacidade para poder resolver. [...] muitas das vezes, acionando o próprio secretário da saúde do Estado, falando diretamente para que possa intervir. Isso não deveria acontecer, porque as vias são claras, mas não funcionam.". (G/HU)

"Quando tem uma demanda inicial, isso é encaminhado para o hospital e à direção do ambulatório. Às vezes o contato é com a secretaria e isso passa pelo setor de faturamento. Eu faço o contato com os profissionais e eles encaixam na agenda a cirurgia, ou a consulta.". (R/HU)

\title{
Tensionamento por parte do controle social
}

Os resultados obtidos na Evidência 1, UIA 1, quanto ao tensionamento por parte do controle social: No $\mathrm{CH}$, este tipo de tensionamento não foi identificado nos relatos dos participantes do estudo. Em SVP é identificado conforme relato do próprio controle social.

"[...] para o hospital do coração em Rio Grande, eu cheguei na casa de uma amiga e disse: "Agora de tarde eu vou lá na secretaria para ver". 'Já me ligaram, tá aqui, já marcaram para daqui a duas semanas'. [...] conseguiram tão rápido, eu fico impressionada.". $(\mathrm{CS} / \mathrm{SVP})$

Em SJN não foi identificado nos relatos dos participantes do estudo. E no RG, este tensionamento é identificado somente no relato do serviço de regulação que interpreta a atuação do controle social conforme evidenciado em sua fala.

"[...] o conselho de saúde atua mais no controle, mas às vezes solicita para agilizar a consulta.". $(R / R G)$

Os resultados obtidos na Evidência 1, UIA 2: No HU, o tensionamento por parte do controle social é identificado conforme relato do serviço de regulação. 
"O conselho tem uma grande importância nesse tipo de atendimento. Eles vêm com o problema, tentam na direção. Passa na direção e eles vêm aqui para o paciente ser atendido. Tentam através do conselho." $(R / H U)$

Os resultados obtidos na Evidência 2 (UIA 2): No HU, o tensionamento por parte do controle social também ocorre via e-mail, e neste sentido foram identificadas quatro mensagens encaminhadas pelo CMS, via e-mail, à direção do HU.

As mensagens versam sobre solicitação de informações quanto a procedimentos cirúrgicos, realização de exames, documentação de referenciamento de usuário para atendimento, solicitação de leitos hospitalares e comunicação de judicialização para obter acesso ao serviço. Segue o teor das mensagens recebidas:

[...] O Formulário em questão não diz respeito à referência ou encaminhamento para outro município. É documento da Defensoria Publica do Rio Grande, necessário a processo movido pela família, naquele órgão, buscando fazer com que o Sistema atenda o [...] com maior agilidade. [...] Aproveito para informar que informarei a família do [...] e vou sugerir aos mesmos que busquem ordem judicial para o preenchimento do documento pelo HU. Cordialmente [...]. (e-mail 6/jul/2015)

[...] Fomos informados que o paciente [...], câncer na região do pescoço se encontra internado no corredor do PA, [...] aguardando por cirurgia. Solicitamos informar qual a estimativa de tempo para a cirurgia e transferência do paciente para leito em quarto. Cordialmente [...]. (e-mail 7/jul/2015)

[...] Foi trazido ao conhecimento desse Conselho que no leito 209-A desse $H U$, se encontra internada [...], com necessidade de leito em UTI, pelo que solicitamos informações tais como: a Central de Leitos a SMS foram acionadas e quais as perspectivas de leitos em UTI na região e ou Estado. Cordialmente [...]. (e-mail 8/abr/2016)

[...] Estamos solicitando informações sobre que relatamos abaixo: [...], relatou-nos que aguarda um Ultra Som das Paredes Abdominais, prescrito pelo [...], desde janeiro/2016. A SMS nos informou que não tem prestador desse serviço e que este exame só e feito no $H U$, por agenda exclusiva do hospital. Se possível gostaríamos de saber se a referida Sra. consta da agenda nesse hospital e qual a previsão de atendimento. Cordialmente [...]. (e-mail 4/mai/2016)

\section{Discussão}

Quanto aos tensionamentos no sistema por ação dos usuários, no $\mathrm{CH}$, a questão da lista de espera e organização da demanda de serviços com deficiência de oferta parece causar uma inconformidade com o sistema, fazendo com que seja acessado um sistema paralelo, ainda que não oficialmente, com o país vizinho, o Uruguai. No entanto, este fluxo também ocorre na direção inversa, em que residentes no Uruguai acessem os serviços no $\mathrm{CH}$.

Em SVP, a população utiliza meios diversificados para verem suas necessidades de saúde atendidas, entre os quais solicitando a intervenção de políticos locais.

A realidade apresentada em ambos os municípios confirma ser a oferta de serviços, por vezes determinada pela pressão que a população exerce sobre a gestão municipal (10).

No entanto, é valido destacar que a dificuldade de gestão possivelmente ocorra pela não possibilidade de dimensionamento, pois os tensionamentos ocorrem por demanda 
inclusive de populações não adscritas, como o que ocorre no $\mathrm{CH}$. Também por intervenção de entes políticos que possuem atuação inclusive na esfera anterior à instalação da necessidade de demanda, em que podem atuar na formulação da política de saúde local, como em SVP.

A realidade encontrada em SVP também se assemelha a reflexão em que a estrutura e as posições dos atores na RAS são capazes de influenciar suas ações, preferências e interpretações de acordo com sua visão de mundo, incluindo os recursos de poder (11).

Em SJN, existe fragilidade na interação entre as equipes de APS e de atenção especializada, e na organização para referências externamente ao município.

Neste particular, é possível perceber tensionamento dos profissionais e da própria população. Esta última tensiona quando considera demorado o fluxo ou insuficiente a assistência e orientações recebidas. Confirmando a importância de que seja verificado como está se dando o processo de marcação de consultas, assim como o tempo de consecução do atendimento, pois desse resultado parte a avaliação do serviço por parte dos usuários (12).

No município do RG, embora o fluxo esteja bom, existem alguns equívocos por parte dos usuários de outros municípios que acessam os serviços de saúde em $\mathrm{RG}$ de forma não convencional.

Estas constatações em ambos os municípios evidenciam que na atenção básica o acesso de usuários ao atendimento organiza-se em torno da oferta das consultas médicas, para as quais existe grande demanda (13), e confirmam resultado de estudo que identifica o fracasso dos fluxos existentes para o acesso aos serviços, com filas de espera para o atendimento pretendido sem que sejam encontradas soluções pelos fluxos preconizados (14).

E que demonstra igualmente o desafio dos profissionais em atender os usuários com suas múltiplas queixas, o que requer uma atenção aquém daquela que a equipe pode oferecer (15).

No HU o tensionamento ocorre no sentido de buscar recursos e assistência de forma mais rápida para o seu problema de saúde, desconsiderando a porta de entrada ao sistema. Este achado corrobora com estudo anteriormente realizado neste mesmo HU, com o objetivo de analisar os fatores determinantes da escolha dos usuários quanto ao serviço de saúde hospitalar, onde os participantes mencionaram que se sentiam acolhidos e com resolutividade para suas necessidades $(16,17)$.

Esta realidade igualmente evidencia nem sempre ser a atenção básica a porta de entrada para o sistema, portanto não exercendo efetivamente o papel de coordenadora da atenção à saúde (10), e que o cotidiano mostra que o pressuposto de existência de uma porta de acesso por meio da rede básica de saúde é fictícia e que na verdade pode até mesmo representar barreiras ao acesso da população aos serviços $(15,17)$.

Quanto aos tensionamentos no sistema exercidos aos e pelos profissionais de saúde, no $\mathrm{CH}$, é utilizado um sistema paralelo com o Uruguai, devido à configuração exclusiva por ser município de fronteira. Este fluxo rompe com a contrarreferência e, embora não tendo sido possível observar neste Estudo de Caso, o tensionamento no serviço de saúde uruguaio é uma questão a ser equacionada.

Esta característica é confirmativa de que a identificação com o processo de trabalho desenvolvido pelos trabalhadores de saúde permite a eles maiores possibilidades de desenvolver alternativas de enfrentamento dos problemas não resolvidos institucionalmente (14). E que os profissionais reconhecem que a organização da RAS em nível de APS ainda é insatisfatório, existindo a necessidade de articulação com os diversos órgãos governamentais para o melhor desempenho da mesma $(18,19)$.

Assim, no cotidiano, os trabalhadores identificam necessidades de usuários, as 
incluem e indicam possíveis alternativas assistenciais e trajetos a serem seguidos na procura de outros serviços $(20,21)$.

Em SVP, os profissionais de saúde, ao perceberem a dificuldade do usuário ao acesso aos serviços por falta de informações sobre o sistema, eles próprios os orientam a procurar a secretaria de saúde para conseguirem atendimento. Fato que confirma a avaliação positiva que recebe a ESF em aspectos relacionados a vínculo (17), orientação para a comunidade e formação profissional voltada para a atenção básica (22).

Ocorre também o contato direto entre profissionais para agilização do fluxo de atendimento. Esta realidade evidencia que o compromisso do profissional para com o usuário resulta num encaminhamento mais adequado às suas necessidades e ainda com mais informações (14).

Em SJN, ainda que com critérios técnicos, existe a prática do contato direto entre profissionais para agilização do fluxo de atendimento. E, em RG, mesmo existindo um fluxo organizado, ainda assim há a necessidade da ação do profissional evidenciando o caso para a efetivação da assistência.

Este achado em nosso estudo vai ao encontro da afirmativa de que a responsabilização pelo acesso do usuário ao serviço é resultado da necessidade pessoal e individual de resolução da demanda e que isso ocorre por meio das redes de relações pessoais, determinando o atendimento, utilizando os contatos pessoais entre os profissionais da rede de saúde, que realizam uma gestão paralela na tentativa de superar as barreiras ao acesso (14). E que a proximidade dos profissionais com as particularidades culturais da comunidade e conhecendo suas condições socioeconômicas, algumas vezes este reconhecimento define a decisão por garantir o atendimento pelo SUS (10).

No HU, o tensionamento possivelmente ocorre por ser este hospital uma referência na região e que, por limitações no quantitativo de instituições para onde encaminhar os pacientes, os profissionais o fazem para o HU. Este achado confirma os resultados do estudo anteriormente realizado neste HU, quando identificou que a rede de saúde era produzida entre e pelos profissionais da medicina, sendo a resolutividade atingida pela queixa-conduta (16).

Quanto ao tensionamento por e entre os gestores: No $\mathrm{CH}$, a questão da lista de espera e organização da demanda de serviços com deficiência de oferta parece causar uma inconformidade com o sistema impulsionando a gestão a tensioná-lo, organizando-se extraoficialmente em redes sociais. Esta realidade confirma que as relações pessoais são capazes de determinar o acesso, o que expõe uma das facetas institucionais na qual o julgamento do grupo implica uma prática de responsabilização pessoal no acesso (14).

Em SVP, na questão da regulação, a gestora relata que acessa os sistemas de regulação, mas que também faz contato direto com outros gestores municipais ou encaminha levando em consideração a prioridade da assistência sobre a organização do sistema.

Esta constatação demonstra a resposta humana da equipe de regulação, com as necessidades e angústias da população. $\mathrm{O}$ processo de responsabilização insere-se no próprio processo de trabalho do gestor de forma natural decorrente da frequência em que ocorre, configurando-se, no entanto, em uma evidente demonstração da insuficiência e incapacidade de resposta do sistema (14). E que os esforços governamentais de expansão de coberturas são contingenciados pelo uso concorrente de recursos (23).

Em SJN, foi possível identificar a fragilidade no referenciamento, com destaque para a vulnerabilidade da gestante de alto risco. Entre as dificuldades apresentadas encontra-se a de regulação pelo não cumprimento da PPI e que em decorrência disso foi feito acordo com outro município que não o da pactuação. E, nesta mesma direção, funcionários da regulação procuram formas de relacionamento dentro do sistema para melhorar o fluxo 
com base nos riscos para organizar a demanda de serviços.

Esta ação do serviço de regulação reafirma a necessidade de integração da rede de atenção, desde a APS à especializada e hospitalar, sendo a plena funcionalidade das centrais de regulação uma das principais estratégias identificadas para essa integração (24). Esta característica é peculiar aos pequenos municípios nos quais se apresentam as maiores dependências externas quanto à capacidade em garantir a integralidade e a equidade em saúde para seus cidadãos gerando tensão permanente entre os diversos atores envolvidos (10).

Em RG, a relação do serviço de regulação da sua secretaria da saúde com as centrais de regulação de outros municípios ocorre na maioria dos casos com a utilização de sistemas informatizados e seguindo contratualização regional. No entanto, também são realizados contatos por e-mails. Há também relato de dificuldades quanto ao acesso aos demais níveis de atenção à saúde, quando referenciados pela atenção básica. Embora esta dificuldade esteja mais relacionada a algumas especialidades, a fragilidade é apontada com destaque para as de maior demanda.

As práticas da regulação do acesso contemplam as diversas faces da ação profissional e perpassam diversos aspectos operacionais da atividade, sendo este um dos efeitos da relação entre os poucos recursos, a alta demanda e o mau funcionamento das rotinas de trabalho. Esta realidade pode gerar iniciativas pessoais de responsabilização e atenção às demandas dos usuários (14).

As centrais de regulação buscam as melhores alternativas assistenciais, mas diante da incapacidade dos mecanismos formais, organizativos e pactuados responderem às necessidades do cotidiano, outros caminhos, mesmo que não preconizados, são utilizados para regulação. Caminhos estes percorridos para permitir o acesso dos usuários ao sistema (14).

Outro tensionamento que também ocorre, embora não por ou entre os gestores, é a descontinuidade do fluxo de informações entre os níveis de atenção à saúde, onde a informação fragmentada ocasiona a perda da consulta por parte do usuário. Tal como no $\mathrm{HU}$, onde o tensionamento ocorre seja pelos desencontros nos sistema de referência e contrarreferência, seja pelo fluxo interno da instituição que, uma vez acessada, dá continuidade ao solicitado nos diversos níveis.

Nesta configuração vale destacar que a prestação de serviços especializados, na maioria das vezes, é realizada em ambiente hospitalar ou em consultórios particulares. Estas duas realidades trazem algumas dificuldades para a atenção longitudinal ao usuário. Quanto a primeira, a dificuldade se apresenta em relação ao estabelecimento e manutenção de vínculo entre este e as equipes da ESF, pois há pouca comunicação entre os profissionais dos diferentes níveis da rede (10). Em relação a segunda, o sistema público coexiste com os serviços privados que se estruturam sob a premissa do consumo de saúde e não a do direito à saúde (25), dicotomia essa que interfere significativamente no acesso aos serviços de saúde.

Quanto ao tensionamento por parte do controle social, no $\mathrm{CH}$ e em SJN, não foi identificado esse tipo de tensionamento. Em SVP, a lista de espera e organização da demanda de serviços parece ser uma dificuldade a ser equacionada, sendo possível identificar a atuação do controle social neste processo quanto a sua interlocução com o município de referência.

Em RG, o serviço de regulação da secretaria municipal da saúde atua utilizando sistemas informatizados, e seguindo contratualização regional. No entanto, o controle social faz contato solicitando urgência no atendimento da demanda.

O tensionamento por parte do controle social, conforme relato do serviço de regulação do HU, ocorre na tentativa de assegurar o cumprimento das diretrizes do SUS e das 
políticas públicas da saúde. Ação esta de forma participativa, mas não como foro de discussão das políticas públicas de saúde.

O tensionamento por parte do controle social ao HU também ocorre via e-mail (Evidência 2), sendo possível verificar que a intenção do CMS é a de agilizar, e de alguma forma prestar esclarecimentos para os usuários que procuram aquele espaço de representatividade. Ato diretamente relacionado com a competência do controle social que é a de fiscalizar e acompanhar o desenvolvimento das ações e dos serviços de saúde e encaminhar os indícios de denúncias aos respectivos órgãos, ocupando de modo pleno os diversos espaços de participação social (26).

\section{Considerações}

Entre as características das RASs estudadas, verificou-se a existência de tensionamentos quanto ao acesso aos serviços de saúde, seja por parte da gestão, dos profissionais de saúde, dos usuários ou do controle social.

Em SJN e RG, é possível perceber que o tensionamento ocorre em decorrência na intenção de buscar alternativas para minimizar as dificuldades de acesso às especialidades e que estas ações se manifestam por contatos telefônicos ou tratativas dos profissionais com a gestão.

No município do $\mathrm{CH}$, a questão do acesso a um sistema paralelo com o país vizinho, o Uruguai, fluxo este que também ocorre na direção inversa, em que residentes no Uruguai acessam os serviços no $\mathrm{CH}$, pode contribuir para a desorganização do sistema, ocasionando dificuldades no planejamento. Em SVP, a população solicita a intervenção de políticos locais para verem suas necessidades de saúde atendidas. Em ambos os municípios, estes casos onde os pontos da rede não se comunicam adequadamente refletem decisivamente na assistência.

No HU, o tensionamento possivelmente ocorra pela questão do seu ambulatório ser visualizado pelo usuário como porta de entrada à atenção especializada e a ele recorrer fazendo contado direto com os profissionais, que uma vez requisitados pelo usuário, por sua vez também reproduzem o efeito de tensionamento no fluxo interno da instituição. Os resultados obtidos na Evidência 2, UIA 2, confirmam que no HU o tensionamento por parte do controle social ocorre inclusive via e-mail.

Consideramos que, com a realização deste estudo de Casos Múltiplos Integrados, é possível afirmar que os tensionamentos como forma de acessar o serviço de ambulatório do HU existem e que ainda faz-se necessário uma efetiva discussão da rede de atenção à saúde da microrregião, não somente na media complexidade, mas por completo, iniciando na APS em busca da integralidade da assistência.

\section{Referências}

1. Mendes EV. As redes de atenção à saúde. Brasília: Organização Pan-Americana da Saúde; 2011. 549 p.: il.

2. Sanchez RM, Ciconelli RM. Conceitos de acesso à saúde. Rev Panam Salud Publica 2012; 31(3): 260268.

3. Starfield B. Atenção Primária. Equilíbrio entre necessidades de saúde, serviços e tecnologia. $2^{\mathrm{a}}$ ed. Brasília (DF): MS; 2004.

4. Rocha KB, Rodríguez-Sanz M, Pasarín MI, Berra S, Gotsens M, Borrell C. Assessment of primary care in health surveys: a population perspective. Eur J Public Health 2012; 22(1):14-19.

5. Conselho das Secretarias Municipais de Saúde do Rio Grande do Sul. Seminário AB 2014. Gestores municipais da saúde debateram o fortalecimento da AB. Revista COSEMSRS 2014; 7:21-33.

6. Fundação de Economia e Estatística. FEEDADOS. Unidades geográficas [Internet]. 2015 [citado em 


\section{Vittalle - Revista de Ciências da Saúde v. 31, n. 2 (2019) 64-76}

2019 mar 14]. Disponível em: http://feedados.fee.tche.br/feedados/\#!home/unidades geograficas/microrregioes/29

7. Costa CFS. O acesso ao ambulatório do hospital universitário da universidade federal do rio grande na rede de atenção à saúde da microrregião litoral lagunar do Rio Grande do Sul: estudo de caso com gestores, profissionais e controle social. Rio Grande/RS. Tese [Doutorado em Enfermagem Saúde] Programa de Pós-graduação em Enfermagem da Escola de Enfermagem da Universidade Federal do Rio Grande; 2016.

8. Yin RK. Estudo de Caso: planejamento e métodos. $4^{\mathrm{a}}$ ed. Porto Alegre (RS): Bookman; 2010, 248p.

9. Moraes R, Galiazzi MC. Análise textual discursiva. 2a ed. Ijuí (RS): Unijuí; 2011.

10. Medeiros CR, Gerhardt TE. Avaliação da Rede de Atenção à Saúde de pequenos municípios na ótica das equipes gestoras. Saúde debate 2015; 39(spe):160-170.

11. Costa CF, Vaghetti HH, Santos SS, Francioni FF, Kerber NP. A complexidade da rede de atenção à saúde. Cienc Cuid Saude 2015; 14 (4): 1609-1615.

12. Reis RS, Coimbra LC, Silva AA, Santos AM, Alves MT, Lamy ZC et al. Acesso e utilização dos serviços na Estratégia Saúde da Família na perspectiva dos gestores, profissionais e usuários. Ciência \& Saúde Coletiva 2013; 18(11): 3321-3331.

13. Silva AM, Mandú EN. Abordagem de necessidades de saúde no encontro assistencial de trabalhadores e usuários na saúde da família Texto Contexto Enferm 2012; 21(4): 739-747.

14. Gawryszewski AR, Oliveira DC, Gomes AM. Acesso ao SUS: representações e práticas de profissionais desenvolvidas nas Centrais de Regulação. Physis Revista de Saúde Coletiva 2012; 22 (1): 119-140.

15. Santos TV, Penna CM. Demandas cotidianas na atenção primária: o olhar de profissionais da saúde e usuários. Texto Contexto Enferm 2013; 22 (1): 149-156.

16. Lemões MA, Costa CF, Ubessi LD, Lange C, Llano PM, Avila JA. Fatores determinantes na escolha dos usuários por serviço hospitalar no acesso à rede de saúde. Revista Contexto \& Saúde Ijuí (RS) 2015; 15(29): 37-44.

17. Gomide MF, Carvalho Pinto IC, Figueiredo LA. Acessibilidade e demanda em uma Unidade de Pronto Atendimento: perspectiva do usuário. Acta Paul Enferm 2012; 25(Número Especial 2): 19-25.

18. Almeida JH, Feitosa AN, Araújo WA, Silva JB, Lourenço LC, Sousa MN. Atenção primária à saúde: enfocando as redes de atenção à saúde. Rev enferm UFPE on line 2015; 9 (11): 9811-9816.

19. Albuquerque MS, Lima LP, Costa AM, Melo Filho DA. Regulação Assistencial no Recife: possibilidades e limites na promoção do acesso. Saúde Soc. 2013; 22 (1): 223-236.

20. Silva AM, Mandú EM, Peduzzi M, Miranda EF. Atuação da enfermagem na abordagem de necessidades de usuários na estratégia saúde da família. Cienc Cuid Saude 2014; 13 (2): 193-201.

21. Assis MM, Jesus WL. Acesso aos serviços de saúde: abordagens, conceitos, políticas e modelo de análise. Ciência \& Saúde Coletiva 2012; 17 (11): 2865-2875.

22. Marin MJ, Marchioli M, Corrente JE. Atenção primária à saúde de uma cidade brasileira sob a ótica dos usuários e profissionais. Cienc Cuid Saude 2015; 14 (3): 1299-1306.

23. Bahia L. Entradas e Bandeiras e o SUS no Século XXI. Ciência \& Saúde Coletiva 2012; 17 (11): 2878-2880.

24. Erdmann AL, Andrade SR, Mello AL, Drago LC. A atenção secundária em saúde: melhores práticas na rede de serviços. Rev Latino-Am Enfermagem [Internet] 2013; 21 (Spec): [08 telas].

25. Santos L, Andrade LO. Acesso às ações e aos serviços de saúde: uma visão polissêmica. Ciência \& Saúde Coletiva 2012; 17(11): 2876-2878.

26. Rolim LB, Cruz RS, Sampaio KJ. Participação popular e o controle social como diretriz do SUS: uma revisão narrativa. Saúde em Debate Rio de Janeiro (RJ) 2013; 37 (96): 139-147. 